\section{Association between corneal \\ biomechanical properties and myopia in Chinese subjects}

Z Jiang ${ }^{1,2,3}$, M Shen ${ }^{1,2}, \mathrm{G}$ Mao $^{1,2,4}$, D Chen ${ }^{1,2}$, $\mathrm{J}^{\mathrm{W}} \mathrm{Wang}^{5}$, J Qu $\mathrm{U}^{1,2}$ and $\mathrm{F} \mathrm{Lu^{1,2 }}$

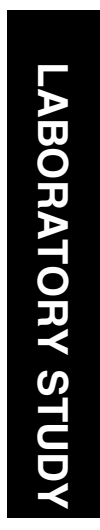

Keywords: corneal biomechanical properties; myopia; central corneal thickness

Purpose To examine the relationship between corneal biomechanical properties and the degree of myopia.

Methods Chinese subjects $(n=172$, age: 11-65 years) were divided into diagnostic groups with non-myopia (spherical equivalence $(S E)>-0.50 \mathrm{D})$, low $(-3.00 \leq \mathrm{SE} \leq-0.50 \mathrm{D})$, moderate $(-6.00 \leq \mathrm{SE}<-3.00 \mathrm{D})$, and high myopia $(\mathrm{SE}<-6.00 \mathrm{D})$. Only the right eye of each subject was analyzed. Central corneal thickness (CCT) was measured by optical coherence tomography. An ocular response analyzer was used to measure corneal hysteresis $(\mathrm{CH})$, corneal resistance factor (CRF), intraocular pressure (IOP), and corneal compensated IOP (IOPcc). Refraction was measured by both automated and subjective refractometry and expressed as SE.

Results $\mathrm{CH}$ was significantly lower in high myopia compared with both low and non-myopia $(P \leq 0.002)$. CCT was 1.5 times more correlated to $\mathrm{CH}$ variation compared with refraction. Similarly, CRF was four times more dependent on CCT than refraction. $\mathrm{CH}$ $(P<0.001)$ or CRF $(P=0.005)$ was positively correlated to refraction. Both IOP and IOPcc were negatively correlated to refraction $(P<0.001)$, respectively.

Conclusions $\mathrm{CH}$ decreases only in high myopia. Refraction is positively correlated to both $\mathrm{CH}$ and CRF but negatively correlated to both IOP and IOPcc. These results indicate that the mechanical strength in anterior segment of the eye is compromised in high myopia. In addition, high myopia may increase the risk of glaucoma. Eye (2011) 25, 1083-1089; doi:10.1038/eye.2011.104; published online 6 May 2011
Myopia is the most common ocular disorder. Myopia prevalence is about 30\% (3-84\%) of people worldwide, with the highest prevalence in East Asians. ${ }^{1-4}$ Its extreme form, high-grade myopia, can increase the risk of glaucoma, retinal detachment, and chorioretinal degeneration. ${ }^{5,6}$ High myopia complications are the fourth leading cause of blindness in England and Wales. ${ }^{7}$

Myopia may be associated with corneal biomechanical properties as well as corneal dimensions such as central corneal thickness (CCT). Several studies have reported the associations between the refractive error and corneal properties, but are still under debate., Chang et $a l^{8}$ demonstrated an association between the refractive error and corneal dimensions and function in Chinese subjects using the ultrasonic pachymeter. In contrast, Fam et $a l^{9}$ reported that CCT measured by the Orbscan II pachymeter had no correlation with myopia in Chinese adults.

Direct clinical measurement of the biomechanical properties of the cornea ${ }^{10}$ has become possible with the recent introduction of a dynamic bi-directional applanation device (ocular response analyzer (ORA); Reichert Ophthalmic Instruments, Depew, NY, USA). ${ }^{11,12}$ Briefly, the ORA is a non-contact tonometer used to measure intraocular pressure (IOP), which obtains information from two corneal applanation pressures that yield values for the biomechanical property of corneal hysteresis $(\mathrm{CH}) . \mathrm{CH}$ is a parameter to measure the corneal

\section{Introduction}

${ }^{1}$ School of Optometry and Ophthalmology and Eye Hospital, Wenzhou Medical College, Zhejiang, China

${ }^{2}$ State Key Laboratory Cultivation Base and Key Laboratory of Vision Science, Ministry of Health PR China and Zhejiang Provincial Key Laboratory of Ophthalmology and Optometry, Zhejiang, China

${ }^{3}$ Department of Ophthalmology, Affiliated First Hospital of Wenzhou Medical College, Zhejiang, China

${ }^{4}$ School of Environmental Science and Public Health, Affiliated First Hospital of Wenzhou Medical College, Zhejiang, China

${ }^{5}$ Bascom Palmer Eye Institute, University of Miami, Miami, FL, USA

Correspondence: F Lu, School of Ophthalmology and Optometry and Eye Hospital, Wenzhou Medical College, 270 Xueyuan Road, Wenzhou, Zhejiang 325027, China

Tel: + 8657788824116 ; Fax: + 8657788824116 E-mail: lufan62@ mail.eye.ac.cn

Received: 2 February 2010 Accepted in revised form: 1 March 2011 Published online: 6 May 2011 
tissue properties that results from viscous damping. ${ }^{11}$ Previous studies reported that $\mathrm{CH}$ was lower in keratoconus, ${ }^{11,13}$ Fuchs' dystrophy, ${ }^{11}$ post laser in situ keratomileusis, ${ }^{11}$ and glaucoma. ${ }^{10}$ The lower $\mathrm{CH}$ was also associated with progressive worsening of the visual field in glaucoma patients. ${ }^{10}$ Using the ORA, additional information such as corneal resistance factor (CRF), and corneal compensated IOP (IOPcc) can be obtained. CRF is an empirically determined parameter that reflects the overall resistance of the cornea. It was demonstrated to be strongly associated with CCT. ${ }^{14}$ IOPcc is a pressure measurement based on data provided by the $\mathrm{CH}$. Results from this measurement are less interfered by corneal properties compared with Goldmann applanation tonometry. ${ }^{14}$

In a previous study, $\mathrm{CH}$ was found significantly lower in high myopic patients (spherical equivalence (SE) $<-9.00 \mathrm{D})$ compared with subjects with the refraction between -3.00 and $0.00 \mathrm{D} .{ }^{15}$ However, several recent ORA studies ${ }^{16-18}$ did not show a correlation between $\mathrm{CH}$ and myopia probably because of the narrow range of myopia selected for the subjects. Therefore, this study re-examined the corneal biomechanical properties in a large sample size of Chinese subjects with the SE from +1.50 to $-29.00 \mathrm{D}$ to determine the relationship between corneal biomechanical properties and myopia.

\section{Subjects and methods}

\section{Subjects}

This experimental study was approved by the Office of Research Ethics Wenzhou Medical College in accordance with the tenets of the Declaration of Helsinki with an informed consent obtained from each subject. A total of 172 Chinese subjects with age ranging from 11 to 65 years (mean \pm SD: $33.12 \pm 12.20$ years) were recruited between March 2006 and June 2007 at the Eye Hospital, Wenzhou Medical College, Wenzhou, Zhejiang, China. Subjects were divided into four diagnostic groups according to refractive status: non-myopia $(\mathrm{SE}>-0.50 \mathrm{D})$, low myopia (SE between -3.00 and $-0.50 \mathrm{D}$ ), moderate myopia (SE between -6.00 and $<-3.00 \mathrm{D}$ ), and high myopia $(\mathrm{SE}<-6.00 \mathrm{D})$. All subjects did not have a history of ocular pathology other than those associated with high myopia.

\section{Instrumentation}

An optical coherence tomography (OCT) system (OCT-3, Carl Zeiss Meditec Inc., Dublin, CA, USA) was used to measure the CCT with the procedures already detailed in our previous studies. ${ }^{15,19-21}$ Briefly, the OCT system was modified by adding a set of lenses on the probe to focus the OCT beam to obtain high quality imaging of the central cornea. The scan width was set at $3 \mathrm{~mm}$ and the actual scan width with the adapter was measured to be $3 \mathrm{~mm}$. To ensure that the central cornea was scanned, all subjects were asked to stare at a fixation target within the OCT system. When the observation and scanning axes were coaxial, the scanning axis was orthogonal to the corneal surface. Under these conditions, a clear reflection was obtained on a monitor with the OCT images recorded. There were 500 axial scans in each image and 1024 pixels in each axial scan.

CCT was obtained from the OCT images using custom software developed at the Wenzhou Medical College, which was similar to previous studies. ${ }^{15,19-22}$ Briefly, central 21 axial scans were aligned by the first peak and averaged to generate a reflectivity profile. The corneal thickness was defied in this profile as the distance between the first and last peaks.

The ORA was used to measure IOP and the $\mathrm{CH}$. Two additional parameters, the IOPCC and CRF were generated by ORA software. In brief, a rapid air puff deformed the cornea and induced corneal bidirectional applanation. An electro-optical system monitored changes in both the inward and outward applanations of the cornea. The $\mathrm{CH}$ was calculated from the difference between two pressures produced by the inward and outward applanations. The average of these two pressures was recorded as the IOP. The CRF and the IOPcc were also calculated from specific combinations of the ORA-induced inward and outward applanation values. The values were derived using proprietary algorithms provided by the manufacturer. ${ }^{14}$

\section{Procedure}

Refraction was measured by both automated and subjective refractometry. Clinical data were obtained, including date of birth, gender, ethnicity, and duration diagnosed as myopia. Only the right eye of each subject was used in this study, and the ORA measurements were taken in triplicates with the mean recorded. To reduce the impact of diurnal variation on the biomechanical properties, all examinations were performed between 1000 and 1700 hours.

\section{Statistics}

Data management and analysis were conducted using Statistical Package for Social Science (version 13.0, SPSS Inc., Chicago, IL, USA). The distribution of the measured variables was estimated by the one-sample KolmogorovSmirnov test. One-way analysis of variance (ANOVA) and post hoc tests were used to determine differences in 
corneal biomechanical properties and CCT among the four diagnostic groups. Multivariate linear regression models were conducted with $\mathrm{CH}, \mathrm{CRF}$, IOP, and IOPCC, as the dependent variables and the relevant predictive factors as covariates for the combined four diagnostic groups. $P$-values $<0.05$ were considered significant.

We certify that all applicable institutional and governmental regulations concerning the ethical use of human volunteers were followed during this research.

\section{Results}

There were no significant differences in $\mathrm{CH}$, IOP, IOPcc, and CRF between the female and male in each group. No correlations were found in the measured parameters with the age.

The refraction among all subjects examined ranged from +1.50 to $-29.00 \mathrm{D}$ (SE). Significant differences in refraction were found between the four diagnostic groups $(P<0.05 ;$ Table 1$)$. The mean age in the high myopic and non-myopic groups was $34.95 \pm 12.17$ and $37.17 \pm 12.17$ years, respectively, which was significantly higher than in the low (26.82 \pm 10.41 years) and moderate myopic ( $24.83 \pm 3.15$ years) groups $(P<0.001$ for each comparison; Table 1). However, there was no significant difference in the gender distribution among the four diagnostic groups ( $\chi^{2}$-test, $P=0.087$; Table 1 ).
There was no significant difference in CCT and CRF among the four diagnostic groups (one-way ANOVA, $P=0.071$ in CCT among four groups and $P=0.682$ in CRF among four groups, respectively; Table 2). The onesample Kolmogorov-Smirnov test showed that $\mathrm{CH}, \mathrm{CRF}$, IOP, or IOPcc was normally distributed, respectively $(P=0.98,0.48,0.52$, and 0.60 , respectively; Figure 1$) . \mathrm{CH}$ in the non-myopic, low, moderate, and high myopic groups were $11.13 \pm 1.45,11.24 \pm 1.47,10.49 \pm 0.89$, and $10.05 \pm 1.66 \mathrm{~mm} \mathrm{Hg}$, respectively (Table 2 ). $\mathrm{CH}$ in the high myopic group was significantly lower than in the low myopic $(P=0.002)$ or non-myopic $(P=0.001)$ group. However, IOP and IOPcc in the high myopic group were significantly higher than in the low myopic and nonmyopic groups $(P=0.035$ and $P<0.001$, respectively).

The regression models showed $\mathrm{CH}$ had $49 \%$ but CRF had $52 \%$ variations. The standardized regression coefficients $(\beta)$ indicated that $\mathrm{CH}$ variation was 1.5 times more dependent on CCT $(\beta=0.631$, Table 3$)$ than refraction $(\beta=0.420)$. Similarly, CRF was four times more dependent on CCT $(\beta=0.710)$ than refraction $(\beta=0.156)$.

There was a low but significantly positive correlation between $\mathrm{CH}$ and refraction $(\beta=0.420, P<0.001$;

Figure 2a) and between CRF and refraction $(\beta=0.156$, $P=0.005$, Figure $2 \mathrm{~b}$ ). For every $1.00 \mu \mathrm{m}$ increase in CCT, the $\mathrm{CH}$ and $\mathrm{CRF}$ increased by 0.031 and $0.039 \mathrm{~mm} \mathrm{Hg}$, respectively, whereas for every $1.00 \mathrm{D}$ increase in refraction, the $\mathrm{CH}$ and $\mathrm{CRF}$ increased by 0.083 and

Table 1 Baseline data of the four diagnostic groups

\begin{tabular}{lccccc}
\hline Parameters & Non-myopia $(\mathrm{n}=65)$ & Low myopia $(\mathrm{n}=34)$ & Moderate myopia $(\mathrm{n}=18)$ & High myopia $(\mathrm{n}=55)$ & $\mathrm{P}$ \\
\hline SE (D) & $0.079 \pm 0.37$ & $-1.53 \pm 0.77$ & $-4.38 \pm 0.87$ & $-6.01 \pm 5.81$ & $<1$ \\
Sex (M/F) & $29 / 36$ & $15 / 19$ & $12 / 6$ & $18 / 37$ & $0.007^{\mathrm{a}}$ \\
Age (years) & $37.17 \pm 12.17$ & $26.82 \pm 10.41$ & $24.83 \pm 3.15$ & $34.95 \pm 12.17$ & $<0.001^{\mathrm{a}}$ \\
\hline
\end{tabular}

Abbreviations: D, diopters; F, female; $\mathrm{M}$, male.

Data were presented as mean \pm SD.

ane-way analysis of variance.

${ }^{\mathrm{b}} \chi^{2}$-test.

Signicant differences in refraction were present among the four diagnostic groups (post hoc tests, $P<0.05$ ). The non-myopic and high myopic subjects were significantly older than the low and moderate myopic subjects.

Table 2 Differences in CCT and corneal biomechanical parameters in the four diagnostic groups

\begin{tabular}{lcccrr}
\hline Parameters & Non-myopia $(\mathrm{n}=65)$ & Low myopia $(\mathrm{n}=34)$ & Moderate myopia $(\mathrm{n}=18)$ & High myopia $(\mathrm{n}=55)$ & P \\
\hline CCT $(\mu \mathrm{m})$ & $524.95 \pm 29.23$ & $536.36 \pm 32.74$ & $520.65 \pm 25.53$ & $536.63 \pm 34.10$ & 0.071 \\
IOP $(\mathrm{mm} \mathrm{Hg})$ & $11.44 \pm 2.72^{* *}$ & $12.23 \pm 3.47^{*}$ & $12.75 \pm 3.98$ & $14.17 \pm 3.24$ & $<0.001$ \\
CH $(\mathrm{mm} \mathrm{Hg})$ & $11.13 \pm 1.45^{* *}$ & $11.24 \pm 1.47^{* *}$ & $10.49 \pm 0.89$ & $8.40 \pm 1.48$ & $10.05 \pm 1.66$ \\
CRF $(\mathrm{mm} \mathrm{Hg})$ & $8.56 \pm 1.60$ & $8.88 \pm 1.74$ & $12.60 \pm 4.31$ & $8.46 \pm 1.98$ & 0.001 \\
IOPcc $(\mathrm{mm} \mathrm{Hg})$ & $10.33 \pm 3.21^{* *}$ & $11.04 \pm 3.94^{* *}$ & $14.72 \pm 3.37$ & $<0.001$ \\
\hline
\end{tabular}

Abbreviations: CCT, central corneal thickness; $\mathrm{CH}$, corneal hysteresis; CRF, corneal resistance factor; IOP, intraocular pressure; IOPcC, corneal compensated IOP.

One-way analysis of variance after post hoc tests; comparison with high myopia group, ${ }^{*} P<0.05$, ${ }^{* *} P<0.01$; statistically significant differences between non-myopic and low myopia compared with high myopic eyes. No significant differences between other diagnostic groups. 

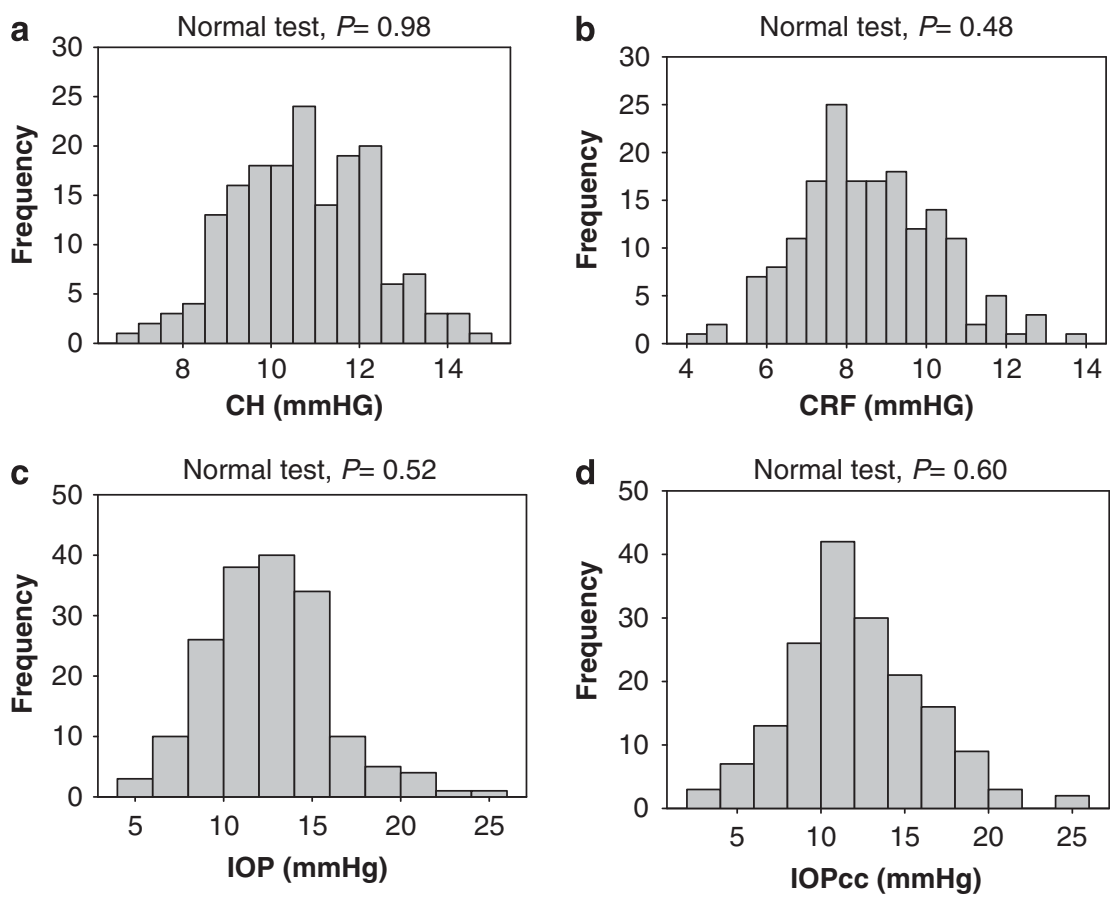

Figure 1 Distributions of corneal hysteresis $(\mathrm{CH})$, corneal resistance factor (CRF), non-contact tonometer intraocular pressure (IOP), and corneal compensated intraocular pressure (IOPcc). On the basis of one-sample Kolmogorov-Smirnov test, (a) CH, (b) CRF, (c) IOP, and (d) IOPcc were normally distributed $(P=0.98,0.48,0.52$, and 0.60 , respectively).

$0.035 \mathrm{mmHg}$, respectively. IOP was correlated to CCT ( $\beta=0.396, P<0.001$; Table 3$)$, but IOPcc was not correlated to CCT after being adjusted with refraction, gender, and age $(\beta=0.028, P=0.695)$. However, both IOP and IOPcC were negatively correlated to the refraction $(\beta \leq-0.280, P<0.001$; Figure 2).

\section{Discussion}

This study investigated correlation of corneal biomechanical properties to the degree of myopia in more details than previous studies. ${ }^{15-18}$ The $\mathrm{CH}$ is similar for non-myopic, low myopic, and moderate myopic eyes, but significantly lower in high myopic eyes compared with the other three groups. These results indicate that $\mathrm{CH}$ is a useful additional assessment for the progression of myopia.

The standardized regression coefficients $(\beta)$ suggest that CCT is 1.5 times more important than refraction in relation to $\mathrm{CH}$. Similarly, $\mathrm{CCT}$ is at least four times more important than refraction in determining $\mathrm{CRF}$ variations. $\mathrm{CH}, \mathrm{CRF}$, and IOP are correlated with CCT. Similar to previous studies, ${ }^{15-18,23}$ IOPcC measurements provided by the ORA are less inuenced by corneal properties than those provided by Goldmann applanation tonometry, ${ }^{23}$ and appears to be a better clinical measurement.
This study shows that both $\mathrm{CH}$ and CRF are correlated to refraction. There is ample evidence that the biomechanical properties of the cornea are correlated to the degree of myopia. First, the elongation of the globe is associated with a flattening cornea and thinning cornea, ${ }^{8}$ resulting in a decrease in $\mathrm{CH}$ and $\mathrm{CRF}{ }^{14,15}$ Second, myopic eyes have a lower ocular rigidity than emmetropic and hyperopic eyes. ${ }^{24,25}$ Therefore in myopia, corneal rigidity, as part of the global rigidity is likely to be low as suggested by the low $\mathrm{CH}$ in this study. Third, according to Kotecha, ${ }^{26}$ corneal biomechanics reflects the viscoelastic properties of the cornea and mechanical strength of stromal collagen fibrils interacting with the extracellular proteoglycan matrix. A significant reduction in the diameter of the scleral collagen fibrils ${ }^{27}$ and the rate of proteoglycan synthesis ${ }^{28}$ is found in the development of myopia, which leads to scleral thinning, the loss of scleral tissue and the weakening of the scleral mechanical properties during scleral remodeling. ${ }^{29}$ Similar changes may occur in the cornea in the development of myopia. ${ }^{22}$

However, other previous studies did not show a correlation between myopia and biomechanical properties of the cornea. ${ }^{16-18}$ This is probably attributed to differences between the present and previous studies in the range of age and/or refractive status, and ethnics for the subjects selected. For instance, in the studies by 
Table 3 Multiple linear regression models of factors associated with IOP, $\mathrm{CH}$, IOPcc, and CRF for the combined four diagnostic groups $^{\mathrm{a}}$

\begin{tabular}{lrrr}
\hline Parameters & $\begin{array}{c}\text { Partial regression Standardized } \\
\text { coefficients }(\beta)\end{array}$ & Poefficients $(\beta)$ \\
\hline CH (adjusted $\left.R^{2}=0.494\right)$ & & & \\
Spherical equivalent & 0.083 & 0.420 & $<0.001$ \\
Central corneal thickness & 0.031 & 0.631 & $<0.001$ \\
Gender & 0.447 & 0.143 & 0.012 \\
Age & -0.010 & -0.080 & 0.152 \\
& & & \\
CRF (adjusted $\left.R^{2}=0.519\right)$ & & & \\
Spherical equivalent & 0.035 & 0.156 & 0.005 \\
Central corneal thickness & 0.039 & 0.710 & $<0.001$ \\
Gender & 0.218 & 0.062 & 0.258 \\
Age & -0.020 & -0.139 & 0.011 \\
& & & \\
IOP (adjusted $\left.R^{2}=0.280\right)$ & & & \\
Spherical equivalent & -0.120 & -0.280 & $<0.001$ \\
Central corneal thickness & 0.042 & 0.396 & $<0.001$ \\
Gender & -0.539 & -0.080 & 0.238 \\
Age & -0.037 & -0.136 & 0.042 \\
& & & \\
IOPcc (adjusted $\left.R^{2}=0.215\right)$ & & & \\
Spherical equivalent & -0.241 & -0.473 & $<0.001$ \\
Central corneal thickness & 0.003 & 0.028 & 0.695 \\
Gender & -1.180 & -0.147 & 0.038 \\
Age & -0.026 & -0.081 & 0.244 \\
\hline
\end{tabular}

Abbreviations: $\mathrm{CH}$, corneal hysteresis; $\mathrm{CRF}$, corneal resistance factor; IOP, intraocular pressure; IOPcc, corneal compensated IOP.

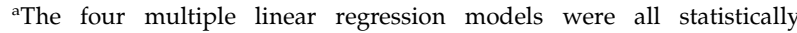
significant $(P<0.001) ; n=172$ for the combined four diagnostic groups.

Lim $e$ e $a^{16}$ and Song et $a l_{,}^{17}$ the subjects are much younger than in the present study and the mean refraction $\left(-2.35 \pm 2.49 \mathrm{D}^{16}-2.20 \pm 1.60 \mathrm{D}^{17}\right)$ is much lower than that in this study $(-5.85 \pm 7.84 \mathrm{D})$. These results from the previous studies support this study that only high myopic eyes have a compromised corneal mechanical strength. ${ }^{30}$ In another study by Fontes et al, ${ }^{18}$ no correlation was found between corneal biomechanical properties and refraction based on Spearman's rank correlation or Pearson's correlation although the subjects have a wide range of refraction $(-19.00$ to $9.00 \mathrm{D})$. However, factors such as age, gender, and CCT have not been adjusted for the results of biomechanical tests in this study. In addition, other factors such as corneal curvature, ${ }^{16}$ race, ${ }^{16}$ axial length, ${ }^{17}$ and retinal vascular caliber ${ }^{31}$ may also influence the correlation between myopia and biomechanical properties of the cornea.

In this study, IOP and IOPcc measured by ORA were negatively correlated to the refraction. These findings are consistent with several previous reports, ${ }^{15,32,33}$ suggesting a positive correlation between IOP and increasing degree of myopia. However, one previous study showed that there was no correlation between IOP and refraction in Chinese children. ${ }^{34}$ Different methods and samples may be the reasons for the inconsistent results of these previous studies and the present study. There is an increased prevalence of glaucoma among myopic eyes compared with non-myopic eyes. ${ }^{32,33}$ However, it is still unclear why myopia causes an increased IOP. One hypothesis is that the increased IOP is related to an increased stress of the global wall and a decreased ocular rigidity in the myopic eye. ${ }^{30}$ According to Schmid et al, ${ }^{30}$ global wall stress $(S)$ is equal to $\operatorname{IOP}^{*}(r / 2 t)$, where $r$ is the inner radius of the globe, and $t$ is the wall thickness, so $\mathrm{IOP}=\left(S^{*} 2 t\right) / r$. In myopic eyes, the inner radius of the globe is longer, and the global wall is thinner compared with non-myopic eyes. If the global wall stress is the same between myopic and non-myopic eyes, the IOP should be lower in myopic eyes according to the formula. However, the IOP measured by ORA and other methods is higher in myopic eyes than in non-myopic eyes. ${ }^{15,32,33}$ Therefore, the results from this study are consistent with the hypothesis of Schmid et al, ${ }^{30}$ that myopia increases IOP by an elevated stress of the global wall and a declined ocular rigidity.

There are some limitations in this study. One limitation of this study is that axial length and corneal curvature were not measured. Therefore, the role of these two factors in the refraction-related mechanical changes of the cornea is unknown. As the non-myopic and high myopic subjects were significantly older than the low and moderate myopic subjects, the age difference between groups may also have a role in the difference of corneal biomechanical properties for these groups. However, the trend between biomechanical properties and refractive development is not affected by the age difference after the adjustment for age, gender, and CCT by multiple linear regression models for all subjects examined. The other limitation is that the investigative groups are not age or gender matched which may influence the results. However, no significant differences were found between the female and male in each group. No significant correlations were found in the measured parameters with the age. These results indicate that age or gender may not influence the results compared with the degree of myopia in this study. In the future, large sample sizes with age and gender match need to be considered in the study design.

In conclusion, refraction is positively correlated to both $\mathrm{CH}$ and CRF but negatively correlated to both IOP and IOPcc. These results indicate that results of corneal biomechanical properties may need to be interpreted in light of the refraction. These results indicate that the mechanical strength in anterior segment of the eye is compromised in high myopia. In addition, high myopia may increase the risk of glaucoma. 
a
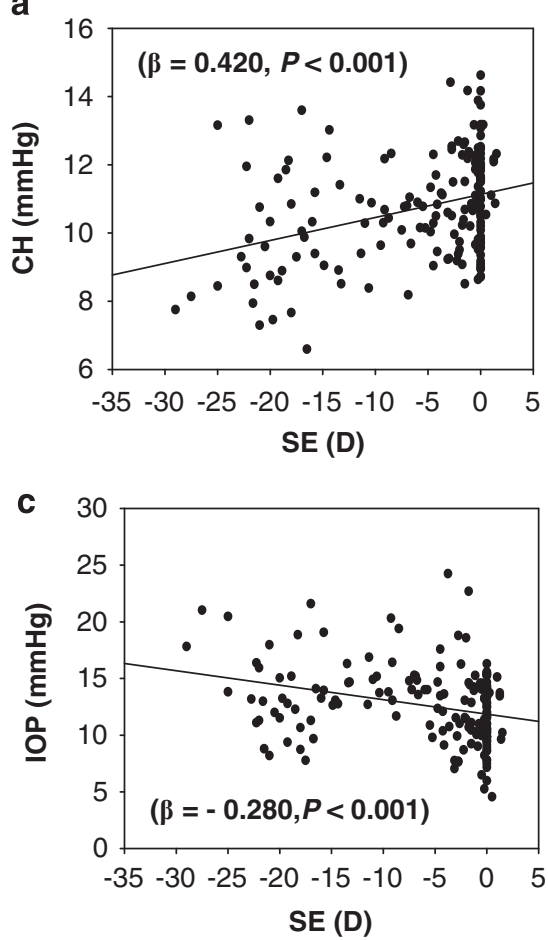

b
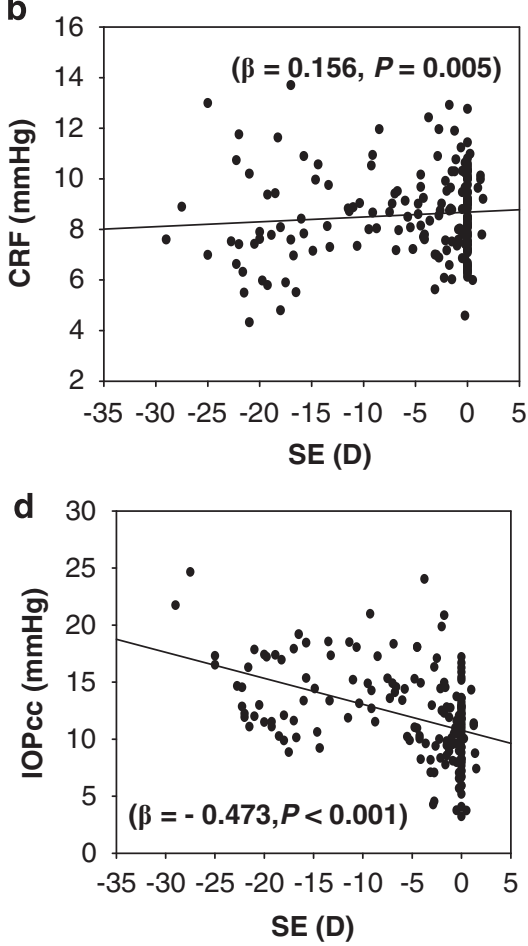

Figure 2 Correlation between spherical equivalent (SE) and corneal hysteresis (CH), CRF (corneal resistance factor), non-contact tonometer intraocular pressure (IOP), and corneal compensated intraocular pressure (IOPcc). There was a weak but significant positive correlation between the refraction and (a) $\mathrm{CH}$ and (b) $\mathrm{CRF}$ (for $\mathrm{CH}, \beta=0.420, P<0.001$; for $\mathrm{CRF}, \beta=0.156, P=0.005$ ). In contrast, the refraction was negatively correlated to (c) IOP and (d) IOPcc (for IOP, $\beta=-0.280, P<0.001$; for IOPcc, $\beta=-0.473, P<0.001$ ).

\section{Summary}

\section{What was known before}

- Several recent ocular response analyzer studies did not show a correlation between corneal hysteresis and myopia probably because of the narrow range of myopia selected for the subjects.

What this study adds

- The purpose of this study was to examine the relationship between corneal biomechanical properties and the degree of myopia.

\section{Conflict of interest}

The authors declare no conflict of interest.

\section{Acknowledgements}

This study was supported by research grants from the Chinese National Science and Technology Development Supporting Program of the Eleventh Five-Year, Beijing, China (2007BAI18B09 to Lu) and the Zhejiang Provincial Program for the Cultivation of High-Level Innovative
Health talents (to Lu). We thank Britt Bromberg, PhD, Xenole Editing, New Orleans, LA, USA, for providing editing services for this manuscript.

\section{References}

1 Feldkamper M, Schaeffel F. Interactions of genes and environment in myopia. Dev Ophthalmol 2003; 37: 34-49.

2 Choo V. A look at slowing progression of myopia. Lancet 2003; 361(9369): 1622-1623.

3 McCarty CA, Taylor HR. Myopia and vision 2020. Am J Ophthalmol 2000; 129(4): 525-527.

4 Fan DS, Lam DS, Lam RF, Lau JT, Chong KS, Cheung EY et al. Prevalence, incidence, and progression of myopia of school children in Hong Kong. Invest Ophthalmol Vis Sci 2004; 45(4): 1071-1075.

5 Paluru P, Ronan SM, Heon E, Devoto M, Wildenberg SC, Scavello $\mathrm{G}$ et al. New locus for autosomal dominant high myopia maps to the long arm of chromosome 17. Invest Ophthalmol Vis Sci 2003; 44(5): 1830-1836.

6 Pararajasegaram R. VISION 2020-the right to sight: from strategies to action. Am J Ophthalmol 1999; 128(3): 359-360.

7 Sorsby A. Blindness in England and Wales. Br Med J 1966; 1(5498): 1251-1252.

8 Chang SW, Tsai IL, Hu FR, Lin LL, Shih YF. The cornea in young myopic adults. Br J Ophthalmol 2001; 85(8): 916-920. 
9 Fam HB, How AC, Baskaran M, Lim KL, Chan YH, Aung T Central corneal thickness and its relationship to myopia in Chinese adults. Br J Ophthalmol 2006; 90(12): 1451-1453.

10 Congdon NG, Broman AT, Bandeen-Roche K, Grover D, Quigley HA. Central corneal thickness and corneal hysteresis associated with glaucoma damage. Am J Ophthalmol 2006; 141(5): 868-875.

11 Luce DA. Determining in vivo biomechanical properties of the cornea with an ocular response analyzer. J Cataract Refract Surg 2005; 31(1): 156-162.

12 Kotecha A, Elsheikh A, Roberts CR, Zhu H, Garway-Heath DF. Corneal thickness- and age-related biomechanical properties of the cornea measured with the ocular response analyzer. Invest Ophthalmol Vis Sci 2006; 47(12): 5337-5347.

13 Shah S, Laiquzzaman M, Bhojwani R, Mantry S, Cunliffe I. Assessment of the biomechanical properties of the cornea with the ocular response analyzer in normal and keratoconic eyes. Invest Ophthalmol Vis Sci 2007; 48(7): 3026-3031.

14 Luce D. Methodology for corneal compensated IOP and corneal resistance factor for an ocular response analyzer. Invest Ophthalmol Vis Sci 2006; 47: E-Abstract 2266.

15 Shen M, Fan F, Xue A, Wang J, Zhou X, Lu F. Biomechanical properties of the cornea in high myopia. Vision Res 2008; 48(21): 2167-2171.

16 Lim L, Gazzard G, Chan YH, Fong A, Kotecha A, Sim EL et al. Cornea biomechanical characteristics and their correlates with refractive error in Singaporean children. Invest Ophthalmol Vis Sci 2008; 49(9): 3852-3857.

17 Song Y, Congdon N, Li L, Zhou Z, Choi K, Lam DS et al. Corneal hysteresis and axial length among Chinese secondary school children: the Xichang Pediatric Refractive Error Study (X-PRES) report no. 4. Am J Ophthalmol 2008; 145(5): 819-826.

18 Fontes BM, Ambrosio Jr R, Alonso RS, Jardim D, Velarde GC, Nose W. Corneal biomechanical metrics in eyes with refraction of -19.00 to $+9.00 \mathrm{D}$ in healthy Brazilian patients. J Refract Surg 2008; 24(9): 941-945.

19 Lu F, Xu S, Qu J, Shen M, Wang X, Fang H et al. Central corneal thickness and corneal hysteresis during corneal swelling induced by contact lens wear with eye closure. Am J Ophthalmol 2007; 143(4): 616-622.

20 Wang J, Fonn D, Simpson TL, Jones L. The measurement of corneal epithelial thickness in response to hypoxia using optical coherence tomography. Am J Ophthalmol 2002; 133(3): 315-319.

21 Wang J, Fonn D, Simpson TL, Jones L. Precorneal and pre- and postlens tear film thickness measured indirectly with optical coherence tomography. Invest Ophthalmol Vis Sci 2003; 44(6): 2524-2528.

22 Muscat S, McKay N, Parks S, Kemp E, Keating D. Repeatability and reproducibility of corneal thickness measurements by optical coherence tomography. Invest Ophthalmol Vis Sci 2002; 43(6): 1791-1795.

23 Medeiros FA, Weinreb RN. Evaluation of the influence of corneal biomechanical properties on intraocular pressure measurements using the ocular response analyzer. J Glaucoma 2006; 15(5): 364-370.

24 Berisha F, Findl O, Fuchsaeger-Mayrl G, Schmetterer L. Dependence of ocular rigidity on eye length: a study comparing ocular pressure pulse and ocular fundus pulse in healthy subjects. Invest Ophthalmol Vis Sci 2004; 45: E-Abstract 2613.

25 Lam AK, Wong S, Lam CS, To CH. The effect of myopic axial elongation and posture on the pulsatile ocular blood flow in young normal subjects. Optom Vis Sci 2002; 79(5): 300-305.

26 Kotecha A. What biomechanical properties of the cornea are relevant for the clinician? Surv Ophthalmol 2007; 52(Suppl 2): S109-S114.

27 McBrien NA, Cornell LM, Gentle A. Structural and ultrastructural changes to the sclera in a mammalian model of high myopia. Invest Ophthalmol Vis Sci 2001; 42(10): 2179-2187.

28 Rada JA, Nickla DL, Troilo D. Decreased proteoglycan synthesis associated with form deprivation myopia in mature primate eyes. Invest Ophthalmol Vis Sci 2000; 41(8): 2050-2058.

29 McBrien NA, Gentle A. Role of the sclera in the development and pathological complications of myopia. Prog Retin Eye Res 2003; 22(3): 307-338.

30 Schmid KL, Li RW, Edwards MH, Lew JK. The expandability of the eye in childhood myopia. Curr Eye Res 2003; 26(2): 65-71.

31 Lim L, Cheung N, Gazzard G, Chan YH, Wong TY, Saw SM. Corneal biomechanical properties and retinal vascular caliber in children. Invest Ophthalmol Vis Sci 2009; 50(1): 121-125.

32 Wong TY, Klein BE, Klein R, Knudtson M, Lee KE. Refractive errors, intraocular pressure, and glaucoma in a white population. Ophthalmology 2003; 110(1): 211-217.

33 Xu L, Wang Y, Wang S, Wang Y, Jonas JB. High myopia and glaucoma susceptibility the Beijing Eye Study. Ophthalmology 2007; 114(2): 216-220.

34 Lee AJ, Saw SM, Gazzard G, Cheng A, Tan DT. Intraocular pressure associations with refractive error and axial length in children. Br J Ophthalmol 2004; 88(1): 5-7. 